ELISAs were performed in triplicate for IL-6 TIMP-1, and MMP-1 levels. In some experiments cells were pretreated with $100 \mathrm{uM}$ of WRW4, a specific formyl peptide receptor inhibitor with A-SAA. Also we used fibroblasts derived from a patient with a genetic non sense mutation that results in no IRAK-4 protein production and hence a halt in TLR signalling. IRAK-4 is a central downstream mediator of Toll-Like Receptor mediated signalling and is crucial for such signalling.

Results Healthy human dermal fibroblasts incubated with A-SAA secreted high levels of IL- 6 compared to untreated control cultures. Moreover A-SAA induced increased levels of TIMP-1 both at the mRNA levels and also the protein levels as determined by ELISA. Levels of the target of TIMP-1, MMP-1 protein levels were not altered at all. Thus the effect leads to a shift in the ratio of TIMP-1 to MMP-1 favouring ECM deposition. Pretreatment with WRW4 prior to A-SAA did not alter IL-6 or TIMP-1 expression levels, showing that the formyl peptide receptor plays no role in TIMP-1 induction mediated by addition of A-SAA. Furthermore cells derived from a gene deleted IRAK-4 patient with no IRAK-4 protein stimulated with A-SAA compared to control fibroblasts had TIMP-1, IL-6 levels compared to non-treated (media alone) dermal fibroblasts.

Conclusions A-SSA induces TIMP-1, but importantly does not alter levels of TIMP-1 target MMP-1, thus shifting the TIMP-1/ MMP-1 ratio. IL-6, a classic proinflammatory cytokine involved in SSc pathogenesis, is also elevated by A-SAA treatment in vitro. The signalling involved IRAK-4, a critical downstream messenger of TLR mediated signalling, but not formyl peptide receptors.

\section{A2.3 ANTI-APOPTOTIC IGG ANTIBODIES FROM PATIENTS WITH PRIMARY SJÖGREN'S SYNDROME AND SYSTEMIC LUPUS ERYTHEMATOSUS INHIBIT THE PHAGOCYTOSIS OF APOPTOTIC CELLS}

doi:10.1136/annrheumdis-2013-203215.3

Aigli G Vakrakou, George E Fragoulis, Menelaos N Manoussakis. Dpt Pathophysiology, University of Athens, Greece

Background and Objectives Recent studies in our laboratory have revealed that a significant portion of SS patients manifests significantly impaired phagocytosis of apoptotic cells (ApoCellphagocytosis), in a manner similar to SLE, a fact which probably leads to the inflammatory and autoimmune responses that characterise these two disorders. Furthermore, our data indicate that approximately $80 \%$ of sera from patients with SS and SLE, but not those with RA and healthy individuals, inhibit the clearance of early apoptotic cells from healthy monocytes. In the present study, we sought to investigate the role of IgG immunoglobulins from patients with SLE and SS in the phagocytosis of apoptotic cells.

Materials and Methods Total IgG immunoglobulin was isolated from the serum of patients with SS $(n=24), \operatorname{SLE}(n=12)$ and RA $(\mathrm{n}=8)$ and healthy donors $(\mathrm{n}=11)$ using Melon Gel Resin columns. Apoptotic Jurkat cells (induction of apoptosis by UV-radiation) were incubated with purified IgG $(50 \mu \mathrm{g} / \mathrm{ml})$ in PBS/BSA $1 \%$. The binding of IgG on the surface of apoptotic cells was assessed by flow cytometry and analysed with the binding index (\% positive cells $\times$ mean fluorescence intensity). ApoCell-phagocytosis was assessed by flow cytometry using monocyte-derived macrophages (MDM) from healthy individuals $(n=3)$ that were incubated $(90 \mathrm{~min})$ with CFSElabelled early apoptotic Jurkat cells which had been opsonised with IgG from patients or healthy individuals or with PBS (control).

Results IgG from patients with SS and SLE exhibited high binding levels in apoptotic cells compared to healthy donors and patients with RA (all for $p<0.0001$ ). Pre-incubation of apoptotic cells with IgG from patients with both SS and SLE had a significant inhibitory effect on the phagocytosis process (comparative ApoCell-phagocytosis: after pre-incubation with PBS $[x=100 \%]$, with IgG from healthy individuals $[\mathrm{n}=11, \mathrm{x}=100 \%]$, with IgG from RA patients $[\mathrm{n}=4$, $\mathrm{x}=100 \%$, with IgG from SS patients $[\mathrm{n}=17, \mathrm{x}=75 \%, \mathrm{p}<0.0001]$, with IgG from SLE patients $[n=10, x=71 \%, p=0.0002])$. Finally, negative correlation was found between the apoptotic cell binding levels of IgG from patients and healthy individuals and the results of ApoCell-phagocytosis assays using apoptotic cells pre-incubated with the corresponding $\operatorname{IgG}(\mathrm{n}=42, \mathrm{p}<0.0001)$.

Conclusions Our results indicate that the impaired phagocytosis of apoptotic cells observed in patients with SS and SLE is primarily due to the presence of antibodies reactive with the surface of apoptotic cells. Further studies need to focus on the mechanism by which immunoglobulins exert such blocking effect, as well as its immunological consequences.

\section{A2.4 ASSOCIATION OF CD55 WITH RETICULAR FIBRES IN THE SYNOVIAL LINING IN RHEUMATOID ARTHRITIS}

doi:10.1136/annrheumdis-2013-203215.4

${ }^{1}$ ON Karpus, ${ }^{2 B}$ Niederreiter, ${ }^{2 P P}$ Tak, $2 \mathrm{JS}$ Smolen, ${ }^{2} \mathrm{HP}$ Kiener, ${ }^{1} \mathrm{~J}$ Hamann. 'Department of Experimental Immunology, Academic Medical Center, Amsterdam, The Netherlands; ${ }^{2}$ Department of Medicine III, Division of Rheumatology, Medical University of Vienna, Vienna General Hospital, Vienna, Austria

Background and Objectives CD55 (decay-accelerating factor) is a complement-regulating protein, expressed at unusually large amounts by fibroblast-like synoviocytes (FLS) in the intimal lining in rheumatoid arthritis (RA). CD55 is also a ligand for CD97, an adhesion-type $G$ protein-coupled receptor, broadly expressed by immune cells invading the inflamed synovium. We previously reported a protective effect of lack of CD55 in experimental models of RA (Hoek et al, Arthritis Rheum 2010; 62(4): 1036-42). Current data cannot explain the high expression of CD55 by FLS. Therefore, we explored in detail the pattern of CD55 expression in RA synovial tissue and 3-D organ cultures.

Materials and Methods Synovial tissue was obtained by miniarthroscopy and analysed by immunohistochemistry staining to visualise CD55 and collagen type III, a constituent of extracellular matrix. Reticular fibres were visualised with Gomori silver staining. Expression of CD55 mRNA in synovial tissue was detected using antisense locked nucleic acid (LNA) oligomers. CD55 expression on 2-D-cultured RA-FLS and on blood cells of healthy individuals was detected by flow cytometry and related to mRNA levels measured by qPCR. 3-D micromasses of RA-FLS were generated in matrigel and analysed by immunohistochemistry for CD55 and reticular fibres.

Results CD55 was highly expressed in the synovial lining of RA tissue, both at the mRNA and the protein level. Notably, CD55 showed an extracellular staining pattern, which coincided with Gomori silver staining of reticular fibres on sequential sections. CD55 expression on 2D-cultured FLS was less abundant and comparable to PBMCs. In 3D-micromasses of RA-FLS, CD55 was upregulated after 3-4 weeks of culture and showed an extracellular distribution that resembled reticular fibres.

Conclusions CD55 mRNA and protein is abundantly expressed by FLS in the intimal lining of RA synovial tissue. We provide evidence that FLS-derived CD55 is deposited in extracellular matrix structures such as reticular fibres, where it may contribute to the synovial stromal address code that facilitates the recruitment of immune cells.

\section{A2.5 CD11c+ DENDRITIC CELLS PLAY AN IMPORTANT PROINFLAMMATORY ROLE IN INFLAMMATORY ARTHRITIS}

doi:10.1136/annrheumdis-2013-203215.5

A Puchner, BS Blüml, V Saferding, B Niederreiter, H Leiss, JS Smolen, K Redlich. Dept of Rheumatology, Medical University of Vienna, Austria

Backround Dendritic cells (DCs) play an important role in bridging the innate and the adaptive immune response by serving as 